\title{
ARTICLE Colon-delivered short-chain fatty acids attenuate the cortisol response to psychosocial stress in healthy men: a randomized, placebo-controlled trial
}

\author{
Boushra Dalile ${ }^{1}{ }^{1}$, Bram Vervliet ${ }^{2}$, Gabriela Bergonzelli ${ }^{3}$, Kristin Verbeke ${ }^{1}$ and Lukas Van Oudenhove ${ }^{1}$
}

Short-chain fatty acids (SCFAs) are products of microbial fermentation of dietary fiber in the colon and may mediate microbiotagut-brain communication. However, their role in modulating psychobiological processes that underlie the development of stressand anxiety-related disorders is not mechanistically studied in humans. In this triple-blind, randomized, placebo-controlled intervention trial, we examine in a parallel group design the effects of 1-week colonic SCFA-mixture delivery in doses equivalent to fermentation of $10 \mathrm{~g}$ or $20 \mathrm{~g}$ of arabinoxylan oligosaccharides on responses to psychosocial stress and fear tasks in 66 healthy men. We demonstrate that low and high doses of SCFAs significantly attenuate the cortisol response to psychosocial stress compared to placebo. Both doses of SCFAs increase serum SCFA levels and this increase in circulating SCFAs co-varies significantly with the attenuation of the cortisol response to psychosocial stress. Colonic SCFA delivery does not modulate fecal SCFA concentrations, serum brain-derived neurotrophic factor, cortisol awakening response, fear learning and extinction, or subjective mood ratings. These results demonstrate that colon-delivered SCFAs modulate hypothalamic-pituitary-adrenal axis reactivity to psychosocial stress, thereby supporting their hypothesized role in microbiota-gut-brain communication.

Neuropsychopharmacology (2020) 45:2257-2266; https://doi.org/10.1038/s41386-020-0732-x

\section{INTRODUCTION}

The gut microbiota may play a critical role in the bidirectional signaling between the gastrointestinal tract and the central nervous system [1]. Microbiota-modifying interventions such as prebiotics and probiotics have demonstrated effects on affective processes, cognition, and behavior in animals, while findings in humans are inconclusive [2-5]. The biological gut-brain signaling mediators driving these effects remain largely unknown. Shortchain fatty acids (SCFAs), microbial metabolites that constitute the major products of bacterial fermentation of dietary fiber in the colon, are considered key candidate mediators in the microbiotagut-brain communication [5]. The most abundant SCFAs are acetate, propionate, and butyrate, which are present in the colon in an approximate molar ratio of 60:20:20, respectively [6]. The amount and relative proportion of individual SCFAs produced in the colon is determined by the type and availability of substrate, gut microbiota composition, and gut transit time [7]. SCFAs function as primary energy sources for luminal colon cells. Beside exerting local effects in the colon, including maintenance of gut barrier integrity, anti-inflammatory and anticarcinogenic effects, SCFAs can mediate microbial signaling to the brain via multiple pathways. SCFAs affect gene expression by inhibiting histone deacetylases (HDACs) [8] and act as endogenous ligands for orphan G-protein coupled receptors [9-11]. SCFAs also affect systemic inflammatory and (gastrointestinal) endocrine responses, can cross the blood-brain barrier (BBB), and signal to the brain via vagal afferents [12-16], thereby interacting with virtually all systems mediating gut-brain communication [5]. Despite knowledge of the multiple pathways that potentially mediate the influence of SCFAs on psychobiological functioning, most research exploring the relationship between SCFAs and brain and behavior comes from rodent studies, is heterogeneous in terms of design and study population, and yields conflicting results. The limited amount of human research is overwhelmingly correlational and heavily relies on quantification of fecal SCFAs [5]. Notwithstanding the inconclusive findings, SCFAs have been implicated both favorably and unfavorably in neuropsychiatric disorders such as Parkinson's disease [17, 18], Alzheimer's disease [19], autism spectrum disorders $[20,21]$, depression $[22,23]$, bipolar disorder [24], and addiction [25], as well as in psychobiological processes including food reward and cognitive function [26] in animals and, to a lesser extent, humans.

Here we adopt a transdiagnostic endophenotype approach by studying the role of SCFAs in two psychobiological affective processes, sensitivity to psychosocial stress and Pavlovian fear conditioning, in healthy subjects rather than a case-control approach comparing patients with healthy controls. These processes reflect activation of the brain's defensive motivational system in response to acute (stress) or potential (anxiety) threat. Stress is the body's attempt to restore homeostasis following

\footnotetext{
${ }^{1}$ Translational Research Center in Gastrointestinal Disorders (TARGID), Department of Chronic Diseases, Metabolism, and Ageing, Faculty of Medicine, KU Leuven, Leuven,

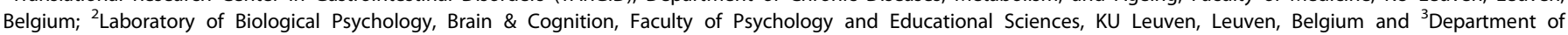
Gastrointestinal Health, Nestlé Research, Société des Produits Nestlé S.A., Lausanne, Switzerland

Correspondence: Lukas Van Oudenhove (lukas.vanoudenhove@kuleuven.be)

These authors contributed equally: Bram Vervliet, Kristin Verbeke, Lukas Van Oudenhove
}

Received: 25 March 2020 Revised: 8 May 2020 Accepted: 1 June 2020

Published online: 10 June 2020 
exposure to a threatening event, but an exaggerated or prolonged stress response can have deleterious effects on mental health [27]. Similarly, learning to fear a threatening event is crucial to the survival of the organism; however, persistent fear of a stimulus that no longer predicts danger can be maladaptive, resulting in pathological levels of anxiety [28, 29]. Both processes help elucidate affective disorder pathophysiology and have been identified as risk factors for the development of affective symptoms and disorders, as well as predictors of treatment response [30-34]. The strong interindividual variability in stress and anxiety responses in healthy humans $[35,36]$ further points to the need to investigate previously unstudied factors such as the role of diet and the hosts' gut microbial composition and function.

To this end, we administered SCFAs or placebo directly to the colon for 1 week and evaluated the effects thereof on psychobiological functioning. We hypothesized that SCFAs would reduce the hypothalamic-pituitary-adrenal (HPA) axis and subjective reactivity to acute psychosocial stress, compared to placebo. We also hypothesized that the SCFA intervention would attenuate fear responses on a computerized task examining acquisition, extinction, and return of fear. We predicted that circulating levels of SCFAs would increase following SCFA intervention relative to placebo, but that no change in fecal SCFA levels would be observed as we postulated quantitative absorption of the SCFAs from the capsules.

\section{MATERIALS AND METHODS}

\section{Subjects}

Sixty-seven Dutch-speaking healthy males with a mean age of $23.6(\mathrm{SD}=2.4)$ and normal $\mathrm{BMI}(\mathrm{M}=22.5, \mathrm{SD}=1.9)$ participated in the study (Table S1). Exclusion criteria is listed in Supplementary Materials and Methods. All participants gave written consent to participate and were compensated for their time. One participant was excluded from data analysis for general noncompliance with experimental procedures. This clinical trial was approved by the Medical Ethics Committee of UZ Leuven/KU Leuven (S60501), conducted in accordance with the Declaration of Helsinki, and preregistered on ClinicalTrials.gov (Title: The Role of Short Chain Fatty Acids in Microbiota-gut-brain Axis (SCFA-AP), Identifier: NCT03688854). The data were collected in the StressLab, located at the Psychiatry division of UZ Leuven, and took place between January and September 2018.

\section{Experimental protocol}

A 1-week, randomized, triple-blind, placebo-controlled trial was performed in a parallel group design, where one group received a low SCFA dose equivalent to 10 grams of arabinoxylan oligosaccharides (AXOS), another group received a high SCFA dose equivalent to 20 grams of AXOS, and a third group received microcrystalline cellulose as placebo [37]. The trial comprised two study visits. Visit 1 was a baseline visit, prior to the commencement of intervention (pre-intervention), and visit 2 was scheduled 1 week later, following the SCFA or placebo intervention (postintervention). In order to standardize fiber intake, all participants adhered to a low-fiber diet starting three days prior to visit 1 up to the end of the trial. Participants kept food diaries for the 3 consecutive days preceding each study visit via www.fatsecret. com (Supplementary Materials and Methods, Table S3). During this period, participants also collected one faecal sample and recorded Bristol stool score prior to each study visit to quantify faecal SCFA levels and stool consistency, respectively.

Each study visit commenced with the collection of saliva samples upon waking for one hour to quantify cortisol awakening response (CAR). This was followed by the consumption of a nofiber breakfast at home on study visit 1 and consuming the same breakfast with the 32 capsules on the morning of study visit 2 . Participants arrived to the laboratory $3 \mathrm{~h}$ later and consumed a standardized no-fiber lunch ad libitum. One hour later, participants completed a battery of questionnaires. Participants then underwent the stress induction procedure followed by a computerized fear learning and extinction task (Fig. S1). Blood samples were collected upon arrival to the lab $(\mathrm{T}+0 \mathrm{~h} 00)$ and in between the psychological tasks $(\mathrm{T}+1 \mathrm{~h} 45, \mathrm{~T}+3 \mathrm{~h} 30$, and $\mathrm{T}+$ 4h45) for analysis of serum SCFAs and serum brain-derived neurotrophic factor (BDNF).

\section{Intervention products}

SCFAs and placebo were delivered directly to the colon using $\mathrm{pH}$ dependent colon-delivery capsules (CDCs). Participants in the high-dose SCFA group consumed capsules containing a SCFA mixture $(174.2 \mathrm{mmol}$ acetate, $13.3 \mathrm{mmol}$ propionate, and 52.4 mmol butyrate) daily. Participants in the low-dose SCFA group consumed 16 capsules of SCFA mixture $(87.1 \mathrm{mmol}$ acetate, 6.6 mmol propionate, and $26.2 \mathrm{mmol}$ butyrate) as well as 16 microcrystalline cellulose capsules. The placebo group consumed 32 microcrystalline cellulose capsules. The amount of SCFA equivalent to $20 \mathrm{~g}$ of AXOS was calculated based on a previous intervention with ${ }^{13} \mathrm{C}$-labelled AXOS [38]. All capsules were coated with a mixture of the polymer Eudragit ${ }^{\circledR}$ FS 30 D (Evonik Industries AG, Darmstadt, Germany) and the antitacking agent PlasACRYL ${ }^{\text {TM }}$ T20 (Evonik Industries AG). In vitro dissolution tests according to the USP XXV paddle method (150 rpm at $37^{\circ} \mathrm{C}$ ) were performed to assess the performance of the capsules. In vivo performance was evaluated using capsules containing ${ }^{13} \mathrm{C}$-labelled SCFA combined with a marker of orocecal transit time as described previously [39].

To promote compliance, participants were allowed to consume the capsules throughout the day and were asked to bring back the non-consumed capsules. All participants reported consumption of all the provided capsules, except one participant who did not consume $0.08 \%$ of the all capsules. No participants dropped out of the study and the capsules were well tolerated.

\section{Psychological readouts}

Stress sensitivity. The Maastricht Acute Stress Test (MAST) [40] consists of a 5-min instruction phase and a 10-min acute stress phase. The acute stress phase comprises multiple trials in which participants immerse their hand in cold $\left(2^{\circ} \mathrm{C}\right)$ water for a period not exceeding $90 \mathrm{~s}$. In between the hand-immersion trials, participants perform a mental arithmetic task, counting backwards from 2043 in steps of 17 or 13 (counterbalanced across the groups and study visits) as quickly and as accurately as possible for at least $45 \mathrm{~s}$ before the next hand-immersion trial starts. To induce social stress, participants were monitored by an experimenter dressed in a white lab-coat, were given negative feedback upon making a mistake, and were 'mock' videotaped throughout the task to later analyze their facial expressions. This task elicits a robust subjective and physiological stress response [40] and does not exhibit significant habituation or sensitization upon repeated administration [41]. To measure cortisol, saliva samples were obtained with synthetic Salivette (Sarstedt AG \& Co., Nümbrecht, Germany) 5 min before (i.e., $t_{\text {pre-stress }(-20 \mathrm{~min})}$ ) and eight times after stress induction (i.e., $t_{+00}, t_{+05}, t_{+15}, t_{+25}, t_{+35}, t_{+45}, t_{+55}$ and $t_{+65}$ $\mathrm{min})$. To measure subjective stress response, participants rated how stressful, painful, and unpleasant the stress induction procedure had been for them on visual analogue scales (VAS) scales (anchors: $0=$ "not at all"; $10=$ "extremely") in the middle, and at the end of the MAST, compared to baseline values.

Fear learning and extinction task. A cue- and context-dependent Pavlovian fear learning and extinction paradigm was employed to investigate the effect of SCFAs on learning and memory processes involved in the etiology of anxiety disorders [42]. In this paradigm, a previously neutral conditioned stimulus (e.g., blue light, CS+) predicted an electrical stimulation (unconditioned stimulus, US) in a given context (in context $\mathrm{A}$ but not in context $\mathrm{B}$ ) while another 
neutral conditioned stimulus (e.g., yellow light, CS-) never predicted an electrical stimulation in either contexts. This was used to induce fear in healthy participants and measure both extinction and return of fear after a lapse of time (recall) or contextual change (renewal) both at pre- and post-intervention. Fear responses were assessed by analyzing skin conductance responses (SCRs) and expectancy ratings of the US (Supplementary Materials and Methods).

Questionnaires. Participants completed a battery of questionnaires during each study visit (Supplementary Materials and Methods). The Positive and Negative Affect Schedule (PANAS) [43] assessed positive and negative emotional states. The Perceived Stress Scale (PSS) [44] assessed the degree to which situations in one's life is appraised as stressful. The Depression, Anxiety, Stress Scales (DASS-21) [45] assessed multiple dimensions of these mood states, including but not limited to dysphoria, hopelessness, situational anxiety, and levels of chronic nonspecific arousal related to stress. Finally, participants also completed the Gastrointestinal Symptom Rating Scale (GSRS) [46], which assessed gastrointestinal symptoms including reflux, abdominal pain, indigestion, diarrhea, and constipation. Participants rated their symptoms and emotional states with "over the past week" as a reference.

\section{Biological readouts}

Analysis of serum SCFA, faecal SCFA, salivary cortisol, and BDNF are described in Supplementary Materials and Methods.

\section{Statistical analysis}

Statistical analysis was conducted using SAS $^{\circledR}$ software version 9.4 (SAS Institute, Cary, NC, USA). Data derived from the stress procedure (cortisol, VAS), fear learning and extinction procedure (US expectancy ratings, SCRs, fear ratings), and biological samples (CAR, serum and faecal SCFA, serum BDNF) were analyzed using linear mixed models (after appropriate transformation of the dependent variables in case of non-Gaussian distribution) with "group" (placebo, low SCFA dose, and high SCFA dose) as betweensubject factor and "visit" (pre- and post-intervention) and, where appropriate, "timepoint" (measurement point within each visit) as within-subject factors, as done previously [47]. This procedure prevented listwise deletion due to missing data. Furthermore, the covariance between test visits is accounted for in our model by choosing an unstructured covariance matrix for the visit variable to allow it to differ between groups. The group-by-visit and the groupby-visit-by-timepoint interaction effects constituted the principal effects of interest. Follow-up planned contrasts were conducted following significant interaction effects $(p$-value $<0.05)$, to test differences of placebo vs. low-dose groups, placebo vs. high-dose groups, and low- vs. high-dose groups. Stepdown Bonferroni adjustment was used to correct for multiple testing comparisons. To investigate whether the changes in circulating SCFA levels were associated with the changes in the cortisol response to stress, we pooled all stress responders $(N=51)$ and entered the change in SCFA levels (post-intervention - pre-intervention) into the linear mixed model as a continuous covariate, including its interaction with visit, while omitting the group variable. Questionnaire data that did not follow Gaussian distribution and could not be transformed were divided into tertiles or quartiles and analyzed using generalized linear mixed models with a cumulative logit link function for ordinal response variables.

Participants' data were excluded from the cortisol analysis if they failed to exhibit a $15.5 \%$ increase from baseline-to-peak in cortisol level at pre-intervention visit [48]. Data were excluded from skin conductance analysis if participants failed to demonstrate successful fear acquisition as indicated by a larger than zero SCRs (CS+ - CS-) during acquisition phase at pre-intervention visit.

\section{RESULTS}

Content of the colon-delivery capsules was released into the systemic circulation in a dose-dependent manner

Consumption of placebo, low or high dose of SCFA had different effects on serum SCFA concentrations as indicated by significant group $\times$ visit interaction effects for total SCFA $(F(2,61)=37.36, p<$ $\left.0.001, \eta_{\mathrm{p}}{ }^{2}=0.366\right)$, acetate $\left(\mathrm{F}(2,61)=34.48, \quad p<0.001, \eta_{\mathrm{p}}{ }^{2}=\right.$ $0.350)$, propionate $\left(F(2,61)=7.67, p=0.001, \eta_{p}{ }^{2}=0.107\right)$, and butyrate $\left(F(2,61)=30.07, p<0.001, \eta_{p}{ }^{2}=0.320\right)$. Planned contrasts revealed that the high-dose group had a greater increase in total SCFA levels $(\mathrm{t}(61)=-8.51, p<0.001, d=2.596)$, acetate ( $\mathrm{t}$ $(61)=-8.16, p<0.001, d=2.489)$, propionate $(\mathrm{t}(61)=-3.23, p=$ $0.004, d=0.985)$, and butyrate $(\mathrm{t}(61)=-7.40, p<0.001, d=2.260)$ relative to the placebo group. Relative to the low-dose group, the high- dose group also had a greater increase in total SCFA levels ( $t$ $(61)=-2.32, p=0.024, d=0.708)$, acetate $(\mathrm{t}(61)=-2.22, p=$ $0.030, d=0.677)$, and butyrate $(\mathrm{t}(61)=-2.23, p=0.029, d=$ $0.680)$ but not propionate $(\mathrm{t}(61)=-0.30, p=0.765)$. Furthermore, the low-dose group had a greater increase in total SCFA levels relative to the placebo group $(\mathrm{t}(61)=3.57, p=0.001, d=1.076)$, as was the case for acetate $(\mathrm{t}(61)=3.43, p=0.002, d=1.034)$, propionate $(\mathrm{t}(61)=3.56, p=0.002, d=1.073)$, and butyrate levels $(\mathrm{t}(61)=5.32, p=0.001, d=1.604)$. These results demonstrate increased systemic SCFA bioavailability following SCFA intervention in a dose-dependent manner for acetate, butyrate, and total SCFA, but not for propionate, for which no significant doseresponse relationship was found (Table 1).

Changes in faecal SCFAs were similar across the groups from pre- to post-intervention (no group $\times$ visit interaction effects for acetate $(\mathrm{F}(2,62)=0.14, p=0.87)$, propionate $(\mathrm{F}(2,62)=0.01, p=$ $0.99)$, butyrate $(F(2,62)=0.21, p=0.81)$, nor total SCFAs $(F(2,62)=$ $0.06, p=0.94)$ ). However, at post-intervention, all fecal SCFAs were lower across all the groups in comparison to pre-intervention (significant main effect of visit for acetate $(F(1,62)=11.29, p=$ $\left.0.001, \eta_{p}{ }^{2}=0.080\right)$, propionate $\left(F(1,62)=6.70, p=0.012, \eta_{p}{ }^{2}=\right.$ $0.049)$, butyrate $\left(F(1,62)=6.70, p=0.010, \eta_{p}{ }^{2}=0.049\right)$, and total SCFAs $\left(F(1,62)=11.10, p=0.002, \eta_{p}{ }^{2}=0.079\right)$ (Table 1$)$.

No effects of the intervention on serum BDNF levels were found in the current study (group $\times$ visit interaction, $F(2,62)=2.27, p=$ 0.11) (Table 1).

Colonic delivery of SCFAs attenuates the cortisol, but not subjective, response to acute psychosocial stress

Only participants who responded to the MAST at pre-intervention visit were included in the cortisol analysis [48], resulting in 17 participants in each treatment group. Consumption of placebo, low or high dose of SCFA had different effects on cortisol response to acute stress (significant group $\times$ visit interaction effect $(F(2,48)$ $=4.76, p=0.013, \eta_{p}{ }^{2}=0.010$, but no group $\times$ visit $\times$ sampletimepoint interaction effect $(\mathrm{F}(16,279)=0.74, p=0.76))$. Follow-up planned contrasts revealed a greater decrease in cortisol levels from pre- to post-intervention in the high dose in comparison with the placebo group $(\mathrm{t}(48)=-2.63, p=0.035, d=0.902)$, as well as in the low dose in comparison with the placebo group $(\mathrm{t}(48)=$ $-2.58, p=0.035, d=0.885$ ) (Fig. 1). There was no dose-effect relationship, as indicated by a lack of a significant difference between the high and low-dose groups $(\mathrm{t}(48)=-0.36, p=0.72)$.

Subjective VAS responses to acute stress revealed no differences between the groups in terms of change from pre- to postintervention on ratings of stress (group $\times$ visit interaction effect $\mathrm{F}(2,63)=0.05, p=0.95)$, pain $(\mathrm{F}(2,63)=0.08, p=0.92)$, nor discomfort $(F(2,63)=1.02, p=0.37)$, neither at baseline, in the middle, nor end of the task (as indicated lack of group $\times$ visit $\times$ timepoint interaction effect for stress $(F(4,125)=1.73, p=0.15)$, pain $(F(4,125)=0.75, p=0.56)$, and discomfort $(F(4,125)=0.61$, $p=0.66$ ) (Fig. S2). An exploratory subgroup analysis was run on the cortisol responders $(N=51)$ (i.e., the sample on which cortisol was analyzed, due to a lack of established cutoff for subjective 
Table 1. Effects of colon-delivered SCFAs on serum SCFAs, faecal SCFAs, and serum BDNF.

\begin{tabular}{|c|c|c|c|c|c|c|c|}
\hline Serum SCFAs $(\mu M)$ & \multicolumn{2}{|l|}{ Placebo } & \multicolumn{2}{|l|}{ Low dose } & \multicolumn{2}{|l|}{ High dose } & $P_{\text {Interactior }}$ \\
\hline Propionate & $1.99(1.58)$ & $1.91(1.49)$ & $2.09(2.16)$ & $2.52(2.61)$ & $1.54(0.87)$ & $2.04(1.54)$ & 0.001 \\
\hline Butyrate & $0.56(0.61)$ & $0.50(0.39)$ & $0.46(0.33)$ & $0.94(0.91)$ & $0.48(0.27)$ & $1.40(1.39)$ & $<0.001$ \\
\hline Faecal SCFAs (mM) & $N=22$ & $N=22$ & $N=22$ & $N=22$ & $N=21$ & $N=21$ & \\
\hline Acetate & $68.21(26.96)$ & $53.96(18.92)$ & $65.84(28.28)$ & 55.17 (20.57) & 72.25 (18.24) & $61.98(17.42)$ & 0.87 \\
\hline Propionate & $16.64(8.78)$ & $13.47(8.97)$ & $18.29(10.33)$ & $14.03(6.33)$ & $18.35(8.80)$ & $14.47(4.98)$ & 0.99 \\
\hline Butyrate & $15.39(7.43)$ & $10.87(3.75)$ & $15.70(11.90)$ & $11.96(4.99)$ & $15.21(7.22)$ & $11.70(6.17)$ & 0.81 \\
\hline Total SCFAs & $100.24(39.78)$ & $78.30(28.83)$ & 99.84 (47.88) & 81.15 (28.28) & $105.82(31.51)$ & $88.15(25.08)$ & 0.94 \\
\hline
\end{tabular}

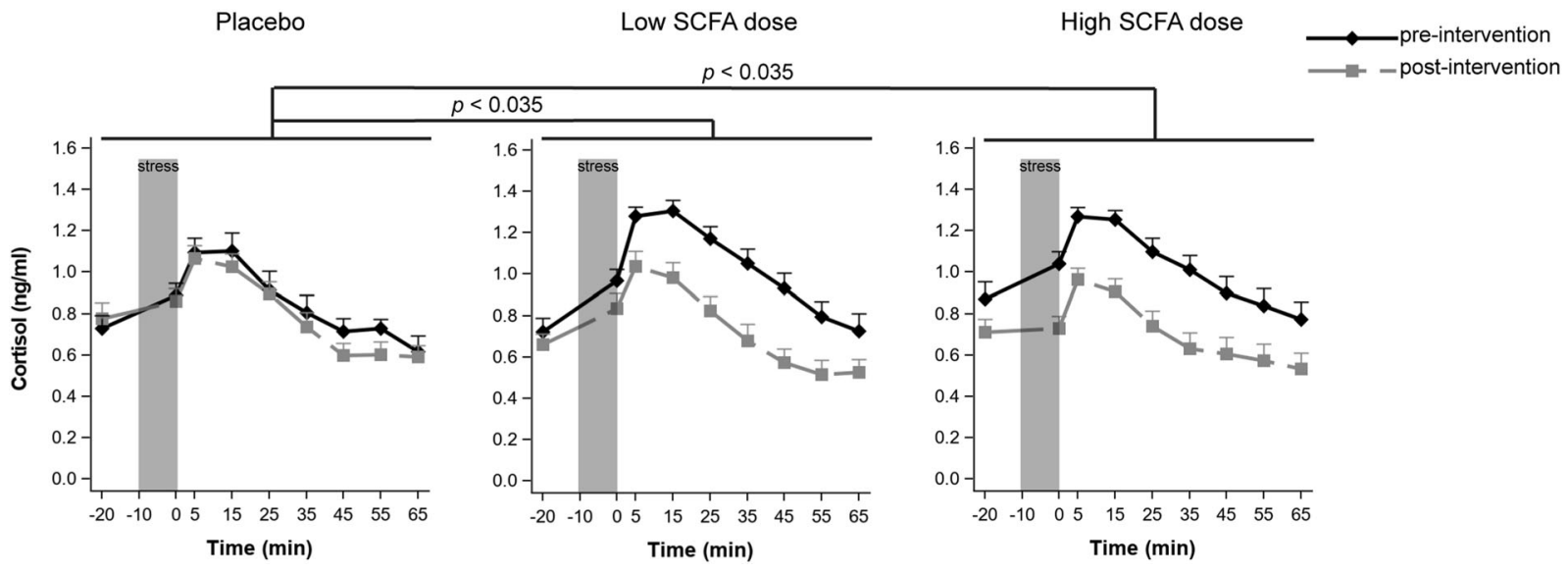

Fig. 1 Effects of colon-delivered SCFAs on cortisol response to acute psychosocial stress. Responses to the Maastricht Acute Stress Test (MAST) at pre- and post-intervention. Cortisol responses to acute psychosocial stress induction procedure. Saliva samples were collected from $20 \mathrm{~min}$ before the stress induction, up to $65 \mathrm{~min}$ after stress induction. Both the low and high SCFA dose groups exhibited significantly greater decreases in cortisol response in comparison to the placebo group $(p=0.035)$. Graphs show means of Box-Cox transformed cortisol (transformed_cortisol $\left.=\left((\text { cortisol }+1)^{* *}-0.5-1\right) /-0.5\right)$ concentrations $\pm S E$.

(non)-response), and is reported in Supplementary Materials and Methods.

Differences in circulating levels of SCFAs co-varied with changes in cortisol levels

We further investigated whether the changes in circulating SCFA levels (as a proxy for absorption of the capsule content) were associated with the changes in the cortisol response to stress. We found that the difference in serum SCFA levels co-varied significantly with the change in cortisol response to acute stress from pre- to post-intervention as indicated by a significant SCFA $\times$ visit interaction effect [acetate $\left(\mathrm{F}(1,811)=11.72, p<0.001, \mathrm{n}_{\mathrm{p}}{ }^{2}=\right.$ $0.012)$, propionate $\left(F(1,811)=4.94, p=0.027, \eta_{p}^{2}=0.005\right)$, butyrate $\left(F(1,811)=7.36, p=0.007, \eta_{p}{ }^{2}=0.008\right)$, and total SCFA $\left.\left(F(1,811)=13.30, p<0.001, \eta_{p}{ }^{2}=0.014\right)\right]$. This indicates that the greater the increase in serum SCFA levels, the greater the decrease in cortisol response to acute stress (Fig. 2).

Cortisol awakening response was not altered by colonic SCFA delivery

Cortisol levels rose upon waking (main effect of timepoint $p<$ 0.001 ). However, the intervention did not affect CAR (group $\times$ visit interaction effect $(F(2,62)=0.49, p=0.62)$, group $\times$ visit $\times$ timepoint interaction effect $(\mathrm{F}(8,245)=1.54, p=0.15))$ (Fig. 3).

Colonic SCFA delivery neither affects psychophysiological nor subjective responses to fear learning and extinction

Fear responses were assessed by analyzing SCRs $(N=53$, Fig. 4a) and expectancy ratings of the US ( $N=66$, Fig. 4b). SCFAs affected neither subjective nor autonomic fear indices over the 


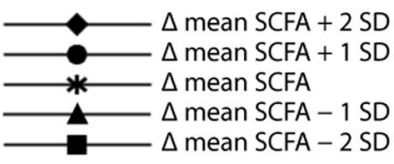

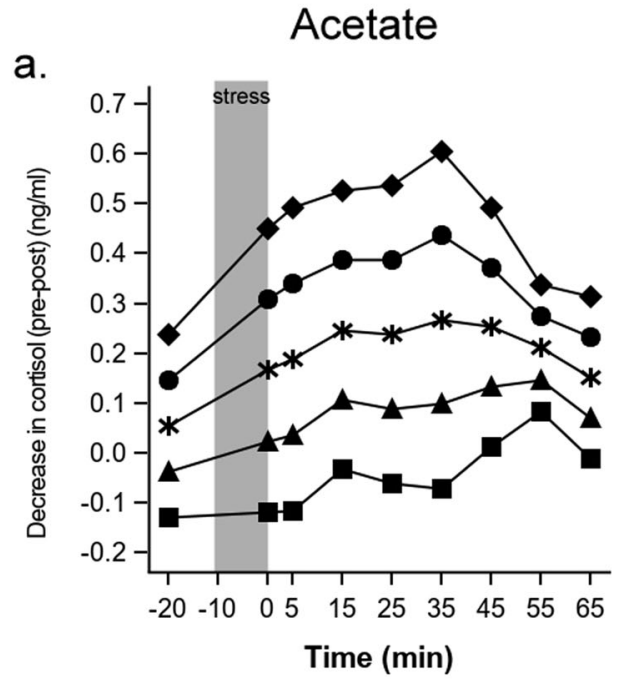

Butyrate

C.

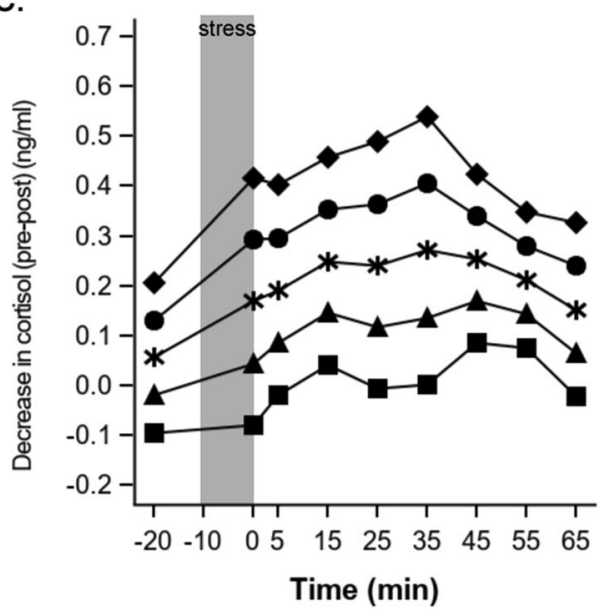

Propionate

b.

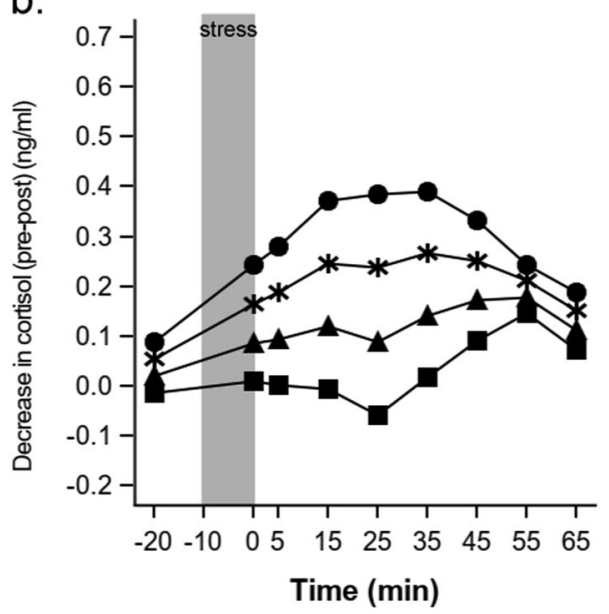

Total SCFAs

d.

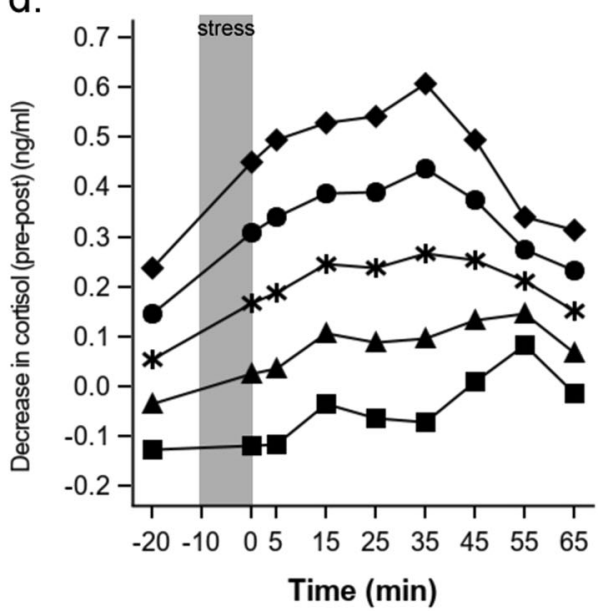

Fig. 2 Associations between changes in serum SCFAs and changes in cortisol response to acute psychosocial stress. Decreases in cortisol levels are plotted in correspondence with mean differential serum SCFA level between -2 and $+2 S D$, representing least to most absorption, respectively. Higher cortisol values represent greater changes in cortisol response to stress from pre- to post-intervention. For a acetate, b propionate, c butyrate, and d total SCFAs, differences in circulating levels of the examined SCFAs co-varied with changes in cortisol levels such that the greater the increase in absorbed SCFAs, the greater the decrease in cortisol response to acute stress.

entire phase of fear acquisition (group $\times$ visit interaction effect SCRs: $(\mathrm{F}(2,50)=0.78, p=0.46)$; US expectancy rating: $(\mathrm{F}(2,60)=1.08, p=$ $0.35)$ ), nor changed the speed of fear acquisition (group $\times$ visit $\times$ trial interaction effect (SCRs: $(\mathrm{F}(8,200)=1.01, p=0.43)$; US expectancy rating $(F(8,240)=0.43, p=1.00))$. Similarly, all groups extinguished the acquired fear to the same extent from pre- to post-intervention (SCRs: $(F(2,47)=1.09, p=0.35)$; US expectancy rating $(F(2,60)=0.42$, $p=0.66)$ ), and at a similar speed when comparing the beginning, middle, and end of extinction phase with the end of acquisition phase within each group (all $p>0.2$ for SCRs and US expectancy rating). There was also no change in fear indices when testing for the return of fear in the extinction context after lapse of time (recall phase) relative to the end of extinction phase (group $\times$ visit $\times$ phase interaction effect SCRs: $(F(2,47)=0.16, p=0.86)$; US expectancy rating: $(F(2,60)=1.45, p=0.24))$. Finally, all groups showed similar levels of fear renewal when presented with the non-reinforced CS+ in the acquisition context (renewal phase), relative to the level of fear displayed during the acquisition phase (with reinforced $\mathrm{CS}_{+}$) (group $\times$ visit $\times$ phase interaction effect SCRs: $\mathrm{F}(2,47)=0.67, p=$ $0.52)$; US expectancy rating: $(F(2,60)=1.46, p=0.24))$.

Colonic SCFA delivery did not alter subjective mood ratings The intervention did not differentially affect subjective ratings on the PANAS (negative affect $(F(2,63)=0.63, p=0.54)$, positive affect $(\mathrm{F}(2,63)=0.65, p=0.53))$, PSS $(\mathrm{F}(2,63)=1.42, p=0.25)$, or the DASS-21 (depression $(F(2,62)=1.49, p=0.23)$, anxiety $(F(2,63)=0.43)$, stress $(F(2,62)=1.26, p=0.29))$. However, the intervention exhibited some effects on the GSRS and Bristol stool chart scores (reported in Supplementary Materials and Methods), but these effects remained within normal ranges. 
Placebo

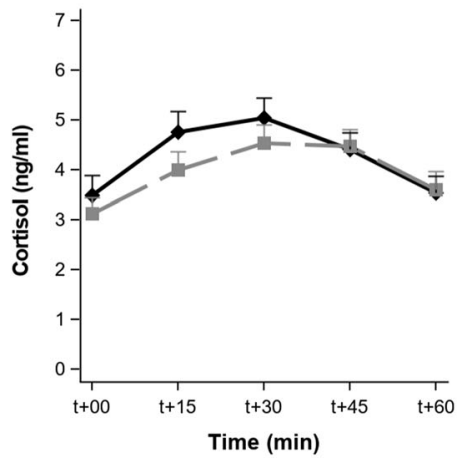

Low SCFA dose

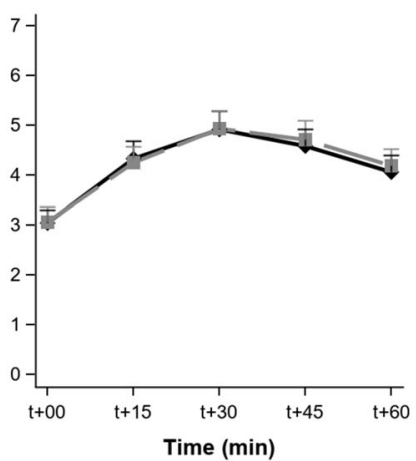

High SCFA dose

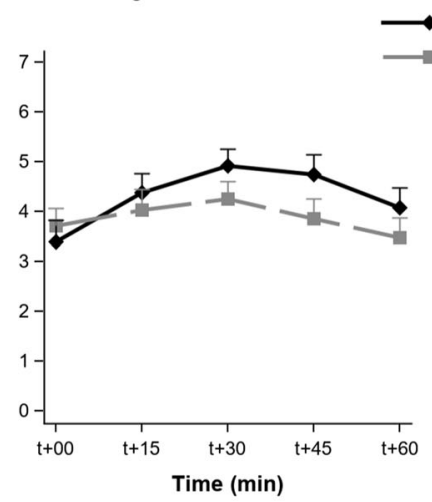

Fig. 3 Effects of colon-delivered SCFAs on cortisol awakening response. Cortisol awakening response (CAR) before and after colonic delivery of placebo, low SCFA dose, and high SCFA dose. CAR was not modulated by colonic SCFA administration. $T+00$ represents time upon waking. Graphs show means of transformed cortisol (transformed_cortisol $\left.=\left((\text { cortisol }+1)^{* *}-0.5-1\right) /-0.5\right)$ concentrations $\pm S E$.

\section{DISCUSSION}

This randomized, triple-blind, placebo-controlled trial in healthy men evaluated for the first time the effect of delivering known amounts of SCFAs into the colon for 1 week on psychobiological outcomes. Colonic administration of physiological doses of SCFAs successfully reached the systemic circulation and attenuated the cortisol response to acute psychosocial stress. Furthermore, the difference in circulating SCFA levels co-varied with changes in cortisol levels, such that the greater the increase in serum SCFAs, the greater the decrease in cortisol response to acute psychosocial stress. SCFAs influenced neither the CAR, processes that underlie the development of fear and anxiety disorders, namely, fear acquisition, extinction, and return of fear, nor subjective mood ratings.

To mechanistically investigate the influence of SCFAs on psychobiological processes, we (a) opted for direct administration of known amounts of SCFAs instead of administering a prebiotic or a dietary fiber. This enables excluding the impact of any other metabolite induced by prebiotic or dietary fiber ingestion; (b) utilized capsules that were coated with a pH-dependent coating to deliver SCFAs directly to the colon, thereby mirroring physiological production and absorption of SCFAs after colonic fermentation. Oral administration of SCFAs (without colon-delivery coating), as is commonly done in animal studies, results in absorption of SCFAs from the upper gastrointestinal tract and may result in different physiological effects. Intravenous administration is nonphysiological as it bypasses the process of absorption and oxidation of SCFAs in the colonocytes. Rectal administration is not suitable for chronic administration. Finally, in contrast to esterified fiber, which additionally releases nonesterified SCFAs, CDCs allow for administration of exact amounts of specific SCFAs; and (c) chose physiological SCFA doses that correspond to those produced after administration of a readily fermentable fiber as determined in our earlier human study [38]. Prebiotic and dietary fiber studies that have evaluated effects on brain function and psychological readouts in humans administered $5-10 \mathrm{~g}$ of fermentable substances daily [49]. To increase the chance of finding an effect while ensuring tolerability of the intervention, we further administered a SCFA dose equivalent to $20 \mathrm{~g}$ of fermentable fiber (AXOS). Taken together, we believe that these methodological aspects facilitated mechanistic investigation of the role of SCFAs in psychological functioning in humans.

We measured serum SCFA concentrations to provide a validation of the CDCs. Serum acetate, propionate, butyrate, and total SCFAs, increased after capsule administration in the low- and high-dose groups, but not in the placebo group. With the exception of propionate, serum concentrations of all SCFAs increased in a dose-dependent manner. The absence of a dose- dependent increase in propionate concentrations can be explained by the low proportion found in the capsules (13.3 $\mathrm{mmol} /$ day). The performance of these capsules was previously evaluated [39] (a) in vitro using a dissolution profile of the CDCs at different $\mathrm{pH}(6.3,6.8,7.0$, and 7.2$)$, and (b) in vivo by simultaneous occurrence of ${ }^{13} \mathrm{CO}_{2}$ (resulting from oxidation of the released ${ }^{13} \mathrm{C}$ labelled SCFA) and ${ }^{14} \mathrm{CO}_{2}$ (marker for orocecal transit time [50]). In the same study, the bioavailability of acetate, propionate and butyrate was determined and accounted for $36 \%, 9 \%$, and $2 \%$ of the administered dose, respectively. In agreement with the proportions found in the capsules, increases in absolute SCFA concentrations in the current study were in the order acetate $(37.62 \mu \mathrm{M} ; 52.07 \mu \mathrm{M})>$ butyrate $(0.48 \mu \mathrm{M} ; 0.92 \mu \mathrm{M})>$ propionate $(0.43 \mu \mathrm{M} ; 0.5 \mu \mathrm{M})$ (low and high dose, respectively). However, the relative increase of acetate was slightly lower (90\% and 163\%) than that of butyrate (104\% and $192 \%)$ due to considerable endogenous production of acetate in the muscles and liver. Together with our previous data, these results confirm that the capsule content was released in the colon and reached the circulation in the expected concentrations.

Colonic administration of SCFAs did not affect fecal SCFA concentrations in our sample, further confirming that the capsules did release SCFAs, which were then efficiently absorbed from the colonic lumen, rather than excreted in feces. The small to medium decrease in fecal SCFAs in all groups between pre- and postintervention visits may be explained by longer adherence to lowfiber diet upon collection of the second fecal sample (at least 7 days) relative to that of the first fecal sample (0-3 days) (Fig. S1). It is possible that due to adherence to low-fiber diet, the availability of substrate to promote SCFA production decreased over time across the groups, which led to lower fecal SCFAs at post-intervention sample. However, it is important to note that fiber intake remained stable from pre- to post-intervention visits across all the groups, and that changes in fecal SCFAs cannot be accounted for by changes in bowel habits (Supplementary Materials and Methods). Previous studies relied on the quantification of fecal SCFAs to perform correlations with neuropsychiatric symptoms or psychological readouts [5]. By standardizing the participants' diets, we attempted to address a limitation in previous gut-brain axis studies, which failed to control for dietary intake in humans when administering probiotics or prebiotics or when correlating fecal SCFAs with psychological functioning or neuropsychiatric symptoms, thereby rendering such associations difficult to interpret [5].

One of the limitations in the field pertains to administering supraphysiological doses of SCFAs to evaluate their effect on brain and behaviour. However, multiple studies suggest that physiological doses of SCFAs can affect brain biology in animals [51-53]. In 

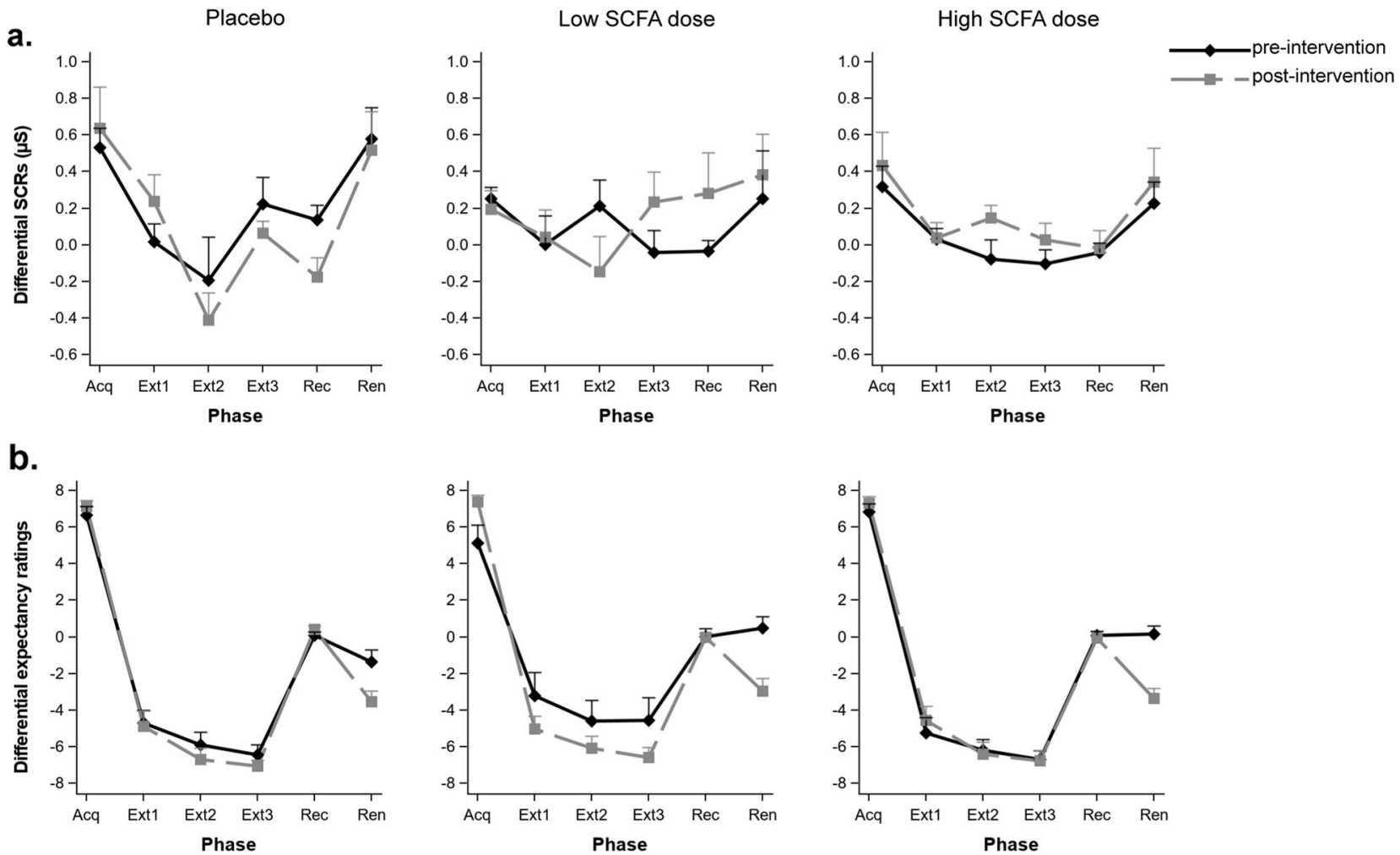

Fig. 4 Effects of colon-delivered SCFAs on fear acquisition, extinction, and return of fear. Psychophysiological and subjective responses to acquisition, extinction (three sub-phases), recall, and renewal of fear at pre- and post-intervention. Neither $\mathbf{a}$ SCRs nor $\mathbf{b}$ expectancy ratings of the unconditioned stimulus (electrical stimulation) were modulated by colonic SCFA administration. SCRs ( $\mu$ S) skin conductance responses in microSiemens, Acq acquisition, Ext extinction, Rec recall, Ren renewal. Graphs show means $\pm S E$.

humans, Byrne et al. [26]. were able to increase SCFA production indirectly by delivering inulin-propionate ester to the colon and observed attenuated neural responses to high-energy food pictures in brain regions involved in reward processing. Our study is the first in humans to deliver exactly known physiological amounts of SCFAs and examine their effects on psychological functioning.

To assess HPA-axis reactivity to experimentally induced psychosocial stress, we administered the MAST as it induces a robust cortisol response and does not exhibit significant habituation or sensitization effects upon repeated testing [41]. Both low and high doses of SCFAs resulted in greater decreases in cortisol response to acute psychosocial stress compared to placebo. Neuroendocrine stress reactivity in animals can be modulated by prebiotic administration [54-56]. However, to date, only two studies investigated the role of SCFAs in HPA-axis reactivity. One study found that in rats, a dose of $1.2 \mathrm{~g} / \mathrm{kg}$ of sodium butyrate acted as a pharmacological stressor, increasing plasma levels of the stress markers corticosterone and adrenocorticotropic hormone (ACTH) [57], whereas a low dose $(200 \mathrm{mg} / \mathrm{kg})$ only slightly increased ACTH. However, butyrate was injected in supraphysiological doses that are typically used to modify histone acetylation, and not in a physiologically-relevant mode of administration. A recent study by Van de Wouw et al. [58]. found that oral administration of SCFAs for 1 week in mice $(67.5 \mathrm{mM}$ acetate, 25 $\mathrm{mM}$ propionate, and $25 \mathrm{mM}$ butyrate) ameliorated stress-induced corticosterone potentiation after an acute stressor. Our results now also indicate that colonic delivery of SCFAs in humans presents one pathway through which changes in colonic microbial activity can affect HPA-axis reactivity to stress. The mechanism through which SCFAs affect HPA-axis reactivity to stress remains to be investigated. Since SCFAs can cross the BBB [59], it is possible to speculate that SCFAs modulate HPA-axis reactivity by penetrating through the BBB via circumventricular organs such as the subfornical organ and the organum vasculosum of the lamina terminalis, which are "leaky" regions of the BBB and are involved in the HPA-axis integration via rich innervations in and projections to the medial parvocellular paraventricular nucleus [60].

In contrast to the cortisol response, subjective ratings collected immediately prior to, in the middle, and upon termination of the acute stress procedure were not modulated by SCFAs. This was contrary to expectations and highlights a mismatch between physiological and subjective stress responses, as previously reported [61, 62]. Stressors that elicit robust physiological responses are not necessarily perceived to be subjectively distressing [62], and significant associations between these two modalities of the stress response were found in only eight out of 30 examined studies (27\%) [61]. This discrepancy may partially be explained by the relatively low levels of stress induced by controlled laboratory procedures in comparison with real life stressful situations [61]. Alternatively, it may be due to the presence of a cognitive component inherent in laboratory stress procedures [63], where, for example, participants may employ external attributions of their psychophysiological reactions that consequently dampens their subjective emotional experience. Of relevance to the present study, higher correspondence between self-reported anxiety and physiological arousal was previously reported in women compared to men [64]. Men might deliberately employ strategies to suppress their emotional expressiveness to protect their self-image, which would subsequently further increase physiological reactivity associated with emotional control $[65,66]$. Similarly, colonic administration of SCFAs did not affect subjective reports of mood and emotional states. This may be explained by the characteristics of our sample, who also exhibited low scores on negative mood at preintervention. This may in turn partially explain why a stress 
challenge was needed to demonstrate the beneficial effect of SCFAs even if the effect was not subjectively evident.

The increase in serum SCFA levels co-varied significantly with the attenuation of the cortisol response to psychosocial stress. This suggests that at least systemic SCFA concentrations may be important to the observed effects, and that circulating acetate, propionate, and butyrate act in the same direction, congruently ameliorating cortisol stress response. Quantification of circulating SCFAs represents an important step towards understanding links between the production of SCFAs and their ultimate biological systemic effects. Previous work in animals has shown that administration of the prebiotics fructo-oligosaccharides (FOS) and galacto-oligosaccharides (GOS) to male rodents increased cecal acetate and propionate concentrations and attenuated stress-induced corticosterone release, and that there was a positive correlation between cecal SCFA levels and reductions in depression- and anxiety-like behavior [54]. Furthermore, prebiotic Bimuno galacto-oligosaccharides (BGOS) increased plasma acetate levels $[67,68]$, cortical GluN2B subunits (involved in glutamate neurotransmission), and acetyl-CoA carboxylase mRNA, all of which also increased following direct administration of acetate [68], suggesting that acetate bioavailability may play a mechanistic role in the observed effects. In sum, these previous studies together with our data indicate that uptake of SCFAs beyond colonic absorption may be critical to their influence on the brain.

In humans, administration of $5 \mathrm{~g}$ /day of BGOS for 3 weeks significantly decreased CAR in healthy male participants [69]. We hypothesized that if subsequent production of SCFAs mediated the effects of prebiotics on CAR, then direct administration of SCFA should elicit the same effect. However, in our study, 1-week colonic administration of SCFAs at a dose equivalent to 10 and 20 $\mathrm{g}$ of AXOS failed to elicit changes in CAR. We chose a shorter intervention period ( 1 week) in comparison with Schmidt et al.'s study [69] as we administered the microbial metabolites directly to the colon, thereby bypassing the need for changes in gut microbiota composition or function, as we assume they are not required to observe the hypothesized effects. The lack of effects on CAR in the present study may alternatively suggest that the effects of the prebiotics were not exclusively mediated by fermentation into SCFAs and increase in their systemic levels. Alternative mechanisms include stimulating the activity and propagation of Bifidobacteria and Lactobacilli in the gut, or catalyzing the release of gut hormones cholecystokinin (CCK), peptide tyrosine tyrosine (PYY) and glucagon-like peptide- 1 (GLP1) [37], or stimulating immune cell activity through Toll-Like Receptor (TLR) dependent signalling [70]. Quantifying additional parameters in future research may better guide such speculations on other microbiota-gut-brain mediators [5].

Responses to experimentally induced fear in humans were examined by assessing fear acquisition, extinction, and return of fear after a lapse of time (recall) or context change (renewal) at the subjective and autonomic level. Fear conditioning is widely regarded as a model to understand pathogenic mechanisms underlying anxiety disorders [71] and is frequently employed in human research. This model is also heavily employed in animal microbiota-gut-brain studies using germ-free and antibiotictreated mice [72], as well as in studies using probiotic [73], prebiotic [54], and butyrate administration in rodents [74], in which freezing behavior is used as an index of anxiety. SCFAs, particularly butyrate, can enhance histone acetylation and thereby affect epigenetic modulation of memory processes underpinning fear, leading to either enhanced initial memory consolidation or facilitating persistent extinction [75]. Previous studies with supraphysiological levels of butyrate or those targeting HDAC inhibition using other pharmacological agents revealed that HDAC inhibitors play an important role in fear learning and extinction processes $[74,76]$. For instance, systemic and intrahippocampal injection of butyrate resulted in persistent fear extinction as indexed by reduced freezing and increased acetylation in the infralimbic cortex $[77,78]$. It is unknown whether physiological doses of butyrate can affect learning and memory processes, particularly in the context of fear extinction. However, a physiological oral dose of butyrate modified brain metabolism and hippocampal neurogenesis in pigs [53]. Furthermore, some studies showed that diet modifies histone acetylation [79] and that SCFAs are at least partially responsible for this modification [80]. However, in the current study, colonic administration of physiological doses of a specific SCFA mix did not affect acquisition, extinction, recall, nor renewal of fear neither at the psychophysiological level (SCRs) nor at the subjective level (predictive ratings of aversive stimulus), similar to previous preclinical work utilizing prebiotics [54].

As an HDAC inhibitor, butyrate increases acetylation around the promoters of neurotrophic factors such as BDNF, thereby increasing their transcription [81, 82]. Furthermore, central and peripheral BDNF are highly correlated in rats $[r=0.86$ ] [83], suggesting that peripheral BDNF can be used as an approximate measure for central BDNF. In humans, whole grain rye bread increased plasma SCFAs [84] and substantially increased (27\%) plasma BDNF concentrations $10.5 \mathrm{~h}$ following ingestion of the product [85]. To gain further understanding on the role of circulating BDNF in the cascade of events mediating the impact of SCFAs on psychobiological functioning, we explored whether circulating BDNF levels were altered after SCFA administration. However, no changes in BDNF concentrations were observed from pre- to post-intervention. This may suggest that beneficial effects on psychobiology are likely mediated by other mechanisms. Alternatively, the amount of SCFAs administered might not have been sufficiently high to modulate circulating BDNF levels.

This study is not without its limitations. First, 15 participants (22.7\%) had to be excluded from analysis of the cortisol response to psychosocial stress due to a lack of stress response at preintervention. However, a post-hoc power calculation revealed that we still had $73 \%$ power with $N=17$ per group to detect a $10-20 \%$ decrease in cortisol following administration of low- and high SCFA doses, respectively. The exclusion of nonresponders also resulted in a nonstatistically significant $(p=0.09)$ numerical imbalance in pre-intervention cortisol levels across the groups. However, we maximally account for this in our statistical model by controlling for pre-intervention differences and allowing the preintervention-post-intervention covariance to vary between groups. Second, blood samples for the quantification of SCFAs were taken only at four timepoints during the test visits, with the baseline sample taken after at least a $2 \mathrm{~h}$ fast. Ideally, to quantitatively detect absorption of SCFAs, a fasted blood sample and frequent sampling is required. This was not possible in our experimental setting due to the timing and duration of the psychological tasks employed. Third, despite successful colonic delivery of SCFAs, the CDCs utilized in this study deliver SCFAs more as a bolus in contrast to gradual SCFA production during colonic fermentation of dietary fiber. Finally, we opted for a homogenous study sample comprising healthy male participants, which particularly limits generalizability to the female population.

Taken together, this study showed that colon-delivered SCFAs attenuate the cortisol response to acute psychosocial stress in healthy male volunteers and may therefore play a mediating role in the psychobiological effects of microbiota-modulating interventions with prebiotics and high-fiber diets. Future work should aim to tease apart the separate effects of colon-delivered acetate, propionate, and butyrate on stress reactivity in humans.

\section{FUNDING AND DISCLOSURE}

The $\mathrm{PhD}$ of $\mathrm{BD}$ is financially supported by an unrestricted grant from Nestlé. GB is an employee of the "Société des produits Nestlé S.A." A patent associated with the results presented in this article 
has been filed. Patent application: 17019-EP-EPA Novel composition for reducing stress disorders. The authors declare no competing interests.

\section{DATA AVAILABILITY}

The ethics protocol and datasets generated and/or analysed during the current study are available from the corresponding author upon reasonable request.

\section{ACKNOWLEDGEMENTS}

We would like to thank Greet Vandermeulen, and Master students Annemarie Hendrix, Geert Spaepen, and Nazlican Tanrioven for assisting during various stages of this trial.

\section{AUTHOR CONTRIBUTIONS}

$\mathrm{BD}, \mathrm{BV}, \mathrm{KV}$, and $\mathrm{LVO}$ designed the study. BD conducted the clinical trial. BD analyzed the results with the help of $B V, K V$, and LVO. BD wrote the manuscript. BD, BV, GB, KV, and LVO revised the manuscript critically and agree to be accountable for all aspects of the work.

\section{REFERENCES}

1. Mayer EA. Gut feelings: the emerging biology of gut-brain communication. Nat Rev Neurosci. 2011;12:453-66.

2. Liu RT, Walsh RFL, Sheehan AE. Prebiotics and probiotics for depression and anxiety: a systematic review and meta-analysis of controlled clinical trials. Neurosci Biobehav Rev. 2019;102:13-23.

3. Wang H, Lee I-S, Braun C, Enck P. Effect of probiotics on central nervous system functions in animals and humans: a systematic review. J Neurogastroenterol Motil. 2016;22:589-605.

4. Reis DJ, llardi SS, Punt SEW. The anxiolytic effect of probiotics: a systematic review and meta-analysis of the clinical and preclinical literature. PloS ONE. 2018;13: e0199041.

5. Dalile B, Van Oudenhove L, Vervliet B, Verbeke K. The role of short-chain fatty acids in microbiota-gut-brain communication. Nat Rev Gastroenterol Hepatol. 2019;16:461-78.

6. Cummings JH, Pomare EW, Branch WJ, Naylor CP, Macfarlane GT. Short chain fatty acids in human large intestine, portal, hepatic and venous blood. Gut. 1987;28:1221-27.

7. Macfarlane S, Macfarlane GT. Regulation of short-chain fatty acid production. Proc Nutr Soc. 2003;62:67-72.

8. Waldecker M, Kautenburger $\mathrm{T}$, Daumann $\mathrm{H}$, Busch $\mathrm{C}$, Schrenk D. Inhibition of histone-deacetylase activity by short-chain fatty acids and some polyphenol metabolites formed in the colon. J Nutr Biochem. 2008;19:587-93.

9. Karaki S-i, Mitsui $R$, Hayashi $H$, Kato I, Sugiya $H$, Iwanaga $T$, et al. Short-chain fatty acid receptor, GPR43, is expressed by enteroendocrine cells and mucosal mast cells in rat intestine. Cell Tissue Res. 2006:324:353-60.

10. Tazoe H, Otomo Y, Karaki S-I, Kato I, Fukami Y, Terasaki M, et al. Expression of short-chain fatty acid receptor GPR41 in the human colon. Biomed Res. 2009;30:149-56

11. Xiong $\mathrm{Y}$, Miyamoto $\mathrm{N}$, Shibata $\mathrm{K}$, Valasek MA, Motoike $\mathrm{T}$, Kedzierski RM, et al Short-chain fatty acids stimulate leptin production in adipocytes through the $G$ protein-coupled receptor GPR41. Proc Natl Acad Sci USA. 2004;101:1045-50.

12. Correa-Oliveira R, Fachi JL, Vieira A, Sato FT, Vinolo MA. Regulation of immune cell function by short-chain fatty acids. Clin Transl Immunol. 2016;5:e73.

13. Tolhurst G, Heffron H, Lam YS, Parker HE, Habib AM, Diakogiannaki E, et al. Shortchain fatty acids stimulate glucagon-like peptide-1 secretion via the G-proteincoupled receptor FFAR2. Diabetes. 2012;61:364-71.

14. Erny D, Hrabe de Angelis AL, Jaitin D, Wieghofer P, Staszewski O, David E, et al. Host microbiota constantly control maturation and function of microglia in the CNS. Nat Neurosci. 2015;18:965-77.

15. Frost G, Sleeth ML, Sahuri-Arisoylu M, Lizarbe B, Cerdan S, Brody L, et al. The short-chain fatty acid acetate reduces appetite via a central homeostatic mechanism. Nat Commun. 2014;5:3611.

16. Goswami C, Iwasaki Y, Yada T. Short-chain fatty acids suppress food intake by activating vagal afferent neurons. J Nutr Biochem. 2018;57:130-35.

17. Unger MM, Spiegel J, Dillmann KU, Grundmann D, Philippeit $H$, Burmann J, et al. Short chain fatty acids and gut microbiota differ between patients with Parkinson's disease and age-matched controls. Parkinsonism Relat Disord. 2016;32:66-72.
18. Sampson T. R., Debelius J. W., Thron T., Janssen S., Shastri G. G., Ilhan Z. E., et al. Gut microbiota regulate motor deficits and neuroinflammation in a model of Parkinson's disease. Cell. 2016;167:1469-80.

19. Ho L, Ono K, Tsuji M, Mazzola P, Singh R, Pasinetti GM. Protective roles of intestinal microbiota derived short chain fatty acids in Alzheimer's disease-type beta-amyloid neuropathological mechanisms. Expert Rev Neurother. 2018;18:83-90.

20. Wang L, Christophersen CT, Sorich MJ, Gerber JP, Angley MT, Conlon MA. Elevated fecal short chain fatty acid and ammonia concentrations in children with autism spectrum disorder. Dig Dis Sci. 2012;57:2096-102.

21. MacFabe DF. Enteric short-chain fatty acids: microbial messengers of metabolism, mitochondria, and mind: implications in autism spectrum disorders. Microb Ecol Health Dis. 2015;26:28177.

22. Szczesniak O, Hestad KA, Hanssen JF, Rudi K. Isovaleric acid in stool correlates with human depression. Nutr Neurosci. 2016;19:279-83.

23. Kelly JR, Borre Y, COB, Patterson E, El Aidy S, Deane J, et al. Transferring the blues: depression-associated gut microbiota induces neurobehavioural changes in the rat. J Psychiatr Res. 2016;82:109-18.

24. Moretti M, Valvassori SS, Varela RB, Ferreira CL, Rochi N, Benedet J, et al. Behavioral and neurochemical effects of sodium butyrate in an animal model of mania. Behav Pharmacol. 2011;22:766-72.

25. Kiraly DD, Walker DM, Calipari ES, Labonte B, Issler O, Pena CJ, et al. Alterations of the host microbiome affect behavioral responses to cocaine. Sci Rep. 2016:6:35455

26. Byrne CS, Chambers ES, Alhabeeb H, Chhina N, Morrison DJ, Preston T, et al. Increased colonic propionate reduces anticipatory reward responses in the human striatum to high-energy foods. Am J Clin Nutr. 2016;104:5-14.

27. Lupien SJ, McEwen BS, Gunnar MR, Heim C. Effects of stress throughout the lifespan on the brain, behaviour and cognition. Nat Rev Neurosci. 2009;10:434-45.

28. Vervliet B, Craske MG, Hermans D. Fear Extinction and Relapse: State of the Art Annu Rev Clin Psychol. 2013;9:215-48.

29. Vervliet B, Raes F. Criteria of validity in experimental psychopathology: application to models of anxiety and depression. Psychol Med. 2013;43:2241-44.

30. LeMoult J, Ordaz SJ, Kircanski K, Singh MK, Gotlib IH. Predicting first onset of depression in young girls: interaction of diurnal cortisol and negative life events. J Abnorm Psychol. 2015;124:850-59.

31. Nederhof E, van Oort FVA, Bouma EMC, Laceulle OM, Oldehinkel AJ, Ormel J. Predicting mental disorders from hypothalamic-pituitary-adrenal axis functioning: a 3-year follow-up in the TRAILS study. Psychol Med. 2015; 45:2403-12.

32. Adam EK, Vrshek-Schallhorn S, Kendall AD, Mineka S, Zinbarg RE, Craske MG. Prospective associations between the cortisol awakening response and first onsets of anxiety disorders over a six-year follow-up-2013 Curt Richter Award Winner. Psychoneuroendocrinology. 2014;44:47-59.

33. Vrshek-Schallhorn S, Doane LD, Mineka S, Zinbarg RE, Craske MG, Adam EK. The cortisol awakening response predicts major depression: predictive stability over a 4-year follow-up and effect of depression history. Psychol Med. 2013;43:483-93.

34. Lenaert B, Boddez Y, Griffith JW, Vervliet B, Schruers K, Hermans D. Aversive learning and generalization predict subclinical levels of anxiety: a six-month longitudinal study. J Anxiety Disord. 2014;28:747-53.

35. MacNamara A, Rabinak CA, Fitzgerald DA, Zhou XJ, Shankman SA, Milad MR, et al. Neural correlates of individual differences in fear learning. Behav Brain Res. 2015;287:34-41.

36. Bogdan R, Nikolova YS, Pizzagalli DA. Neurogenetics of depression: a focus on reward processing and stress sensitivity. Neurobiol Dis. 2013;52:12-23.

37. Daud NM, Ismail NA, Thomas EL, Fitzpatrick JA, Bell JD, Swann JR, et al. The impact of oligofructose on stimulation of gut hormones, appetite regulation and adiposity. Obesity (Silver Spring). 2014;22:1430-38.

38. Boets E. Production of short chain fatty acids in the human colon and their impact on health. Doctoral thesis, KU Leuven, 2016.

39. Boets E, Gomand SV, Deroover L, Preston T, Vermeulen K, De Preter V, et al Systemic availability and metabolism of colonic-derived short-chain fatty acids in healthy subjects: a stable isotope study. J Physiol. 2017;595:541-55.

40. Smeets T, Cornelisse S, Quaedflieg CW, Meyer T, Jelicic M, Merckelbach $H$. Introducing the Maastricht Acute Stress Test (MAST): a quick and non-invasive approach to elicit robust autonomic and glucocorticoid stress responses. Psychoneuroendocrinology. 2012;37:1998-2008.

41. Quaedflieg C, Meyer T, van Ruitenbeek P, Smeets T. Examining habituation and sensitization across repetitive laboratory stress inductions using the MAST. Psychoneuroendocrinology. 2017;77:175-81.

42. Zeidan MA, Lebron-Milad K, Thompson-Hollands J, Im JJ, Dougherty DD, Holt DJ, et al. Test-retest reliability during fear acquisition and fear extinction in humans. CNS Neurosci Ther. 2012;18:313-7. 
43. Watson D, Clark LA, Tellegen A. Development and validation of brief measures of positive and negative affect: the PANAS scales. J Personal Soc Psychol. 1988;54:1063-70.

44. Cohen S, Kamarck T, Mermelstein R. A global measure of perceived stress. J Health Soc Behav 1983;24:385-96.

45. Henry JD, Crawford JR. The short-form version of the Depression Anxiety Stress Scales (DASS-21): construct validity and normative data in a large non-clinical sample. Br J Clin Psychol. 2005;44:227-39.

46. Dimenas E, Glise H, Hallerback B, Hernqvist $H$, Svedlund J, Wiklund I. Well-being and gastrointestinal symptoms among patients referred to endoscopy owing to suspected duodenal ulcer. Scand J Gastroenterol. 1995;30:1046-52.

47. Hoge EA, Bui E, Palitz SA, Schwarz NR, Owens ME, Johnston JM, et al. The effect of mindfulness meditation training on biological acute stress responses in generalized anxiety disorder. Psychiatry Res. 2018;262:328-32.

48. Miller R, Plessow F, Kirschbaum C, Stalder T. Classification criteria for distinguishing cortisol responders from nonresponders to psychosocial stress: evaluation of salivary cortisol pulse detection in panel designs. Psychosom Med. 2013;75:832-40

49. Serra MC, Nocera JR, Kelleher JL, Addison O. Prebiotic intake in older adults: effects on brain function and behavior. Curr Nutr Rep. 2019;8:66-73.

50. Verbeke K, de Preter V, Geboes K, Daems T, van den Mooter G, Evenepoel P, et al. In vivo evaluation of a colonic delivery system using isotope techniques. Alimentary Pharmacol Ther. 2005;21:187-94.

51. Arnoldussen $I A C$, Wiesmann $M$, Pelgrim $C E$, Wielemaker EM, van Duyvenvoorde W, Amaral-Santos PL, et al. Butyrate restores HFD-induced adaptations in brain function and metabolism in mid-adult obese mice. Int J Obes. 2017:41:935.

52. Yang T, Magee KL, Colon-Perez LM, Larkin R, Liao Y-S, Balazic E, et al. Impaired butyrate absorption in the proximal colon, low serum butyrate and diminished central effects of butyrate on blood pressure in spontaneously hypertensive rats. Acta Physiol. 2019;226:e13256.

53. Val-Laillet D, Guérin S, Coquery N, Nogret I, Formal M, Romé V, et al. Oral sodium butyrate impacts brain metabolism and hippocampal neurogenesis, with limited effects on gut anatomy and function in pigs. FASEB J. 2018;32:2160-71.

54. Burokas A, Arboleya S, Moloney RD, Peterson VL, Murphy K, Clarke G, et al. Targeting the microbiota-gut-brain axis: prebiotics have anxiolytic and antidepressant-like effects and reverse the impact of chronic stress in mice. Biol Psychiatry. 2017;82:472-87.

55. Forsatkar MN, Nematollahi MA, Rafiee G, Farahmand H, Lawrence C. Effects of the prebiotic mannan-oligosaccharide on the stress response of feed deprived zebrafish (Danio rerio). Physiol Behav. 2017;180:70-7.

56. Mika A, Day HE, Martinez A, Rumian NL, Greenwood BN, Chichlowski M, et al. Early life diets with prebiotics and bioactive milk fractions attenuate the impact of stress on learned helplessness behaviours and alter gene expression within neural circuits important for stress resistance. Eur J Neurosci. 2017:45:342-57.

57. Gagliano H, Delgado-Morales R, Sanz-Garcia A, Armario A. High doses of the histone deacetylase inhibitor sodium butyrate trigger a stress-like response. Neuropharmacology. 2014;79:75-82.

58. van de Wouw M, Boehme M, Lyte JM, Wiley N, Strain C, O'Sullivan O, et al. Shortchain fatty acids: microbial metabolites that alleviate stress-induced brain-gut axis alterations. J Appl Physiol. 2018;596:4923-44.

59. Mitchell RW, On NH, Del Bigio MR, Miller DW, Hatch GM. Fatty acid transport protein expression in human brain and potential role in fatty acid transport across human brain microvessel endothelial cells. J Neurochem. 2011;117:735-46.

60. Ziegler DR, Herman JP. Neurocircuitry of stress integration: anatomical pathways regulating the hypothalamo-pituitary-adrenocortical axis of the rat1. Integr Comp Biol. 2002;42:541-51

61. Campbell J, Ehlert U. Acute psychosocial stress: does the emotional stress response correspond with physiological responses? Psychoneuroendocrinology. 2012;37:1111-34.

62. Dickerson SS, Kemeny ME. Acute stressors and cortisol responses: a theoretical integration and synthesis of laboratory research. Psychological Bull. 2004;130: 355-91.

63. Mauss IB, Levenson RW, McCarter L, Wilhelm FH, Gross JJ. The tie that binds? Coherence among emotion experience, behavior, and physiology. Emotion. 2005;5:175.
64. Avero P, Calvo MG. Emotional reactivity to social-evaluative stress: genderdifferences in response systems concordance. Pers Individ Differ. 1999;27:155-70.

65. Gross JJ. Emotion regulation: affective, cognitive, and social consequences. Psychophysiology. 2002;39:281-91

66. Lam S, Dickerson SS, Zoccola PM, Zaldivar F. Emotion regulation and cortisol reactivity to a social-evaluative speech task. Psychoneuroendocrinology. 2009;34:1355-62.

67. Kao AC, Spitzer S, Anthony DC, Lennox B, Burnet PWJ. Prebiotic attenuation of olanzapine-induced weight gain in rats: analysis of central and peripheral biomarkers and gut microbiota. Transl Psychiatry. 2018;8:66.

68. Gronier B, Savignac HM, Di Miceli M, Idriss SM, Tzortzis G, Anthony D, et al. Increased cortical neuronal responses to NMDA and improved attentional setshifting performance in rats following prebiotic (B-GOS((R))) ingestion. Eur Neuropsychopharmacol J Eur Coll Neuropsychopharmacol. 2018;28:211-24.

69. Schmidt K, Cowen PJ, Harmer CJ, Tzortzis G, Errington S, Burnet PW. Prebiotic intake reduces the waking cortisol response and alters emotional bias in healthy volunteers. Psychopharmacology (Berl). 2015;232:1793-801.

70. Peshev D, Van den Ende W. Fructans: prebiotics and immunomodulators. J Funct Foods. 2014;8:348-57.

71. Duits P, Cath DC, Lissek S, Hox JJ, Hamm AO, Engelhard IM, et al. Updated metaanalysis of classical fear conditioning in the anxiety disorders. Depress Anxiety. 2015:32:239-53.

72. Chu C, Murdock MH, Jing D, Won TH, Chung H, Kressel AM, et al. The microbiota regulate neuronal function and fear extinction learning. Nature. 2019;574:543-48.

73. Peng $\mathrm{H}-\mathrm{H}$, Tsai T-C, Huang $\mathrm{W}-\mathrm{Y}$, Wu H-M, Hsu K-S. Probiotic treatment restores normal developmental trajectories of fear memory retention in maternally separated infant rats. Neuropharmacology. 2019;153:53-62.

74. Stilling RM, van de Wouw M, Clarke G, Stanton C, Dinan TG, Cryan JF. The neuropharmacology of butyrate: the bread and butter of the microbiota-gutbrain axis? Neurochem Int. 2016;99(Supplement C):110-32.

75. Lattal KM, Wood MA. Epigenetics and persistent memory: implications for reconsolidation and silent extinction beyond the zero. Nat Neurosci. 2013;16:124-9.

76. Whittle N, Singewald N. HDAC inhibitors as cognitive enhancers in fear, anxiety and trauma therapy: where do we stand? Biochemical Soc Trans. 2014;42:569-81.

77. Lattal KM, Barrett RM, Wood MA. Systemic or intrahippocampal delivery of histone deacetylase inhibitors facilitates fear extinction. Behav Neurosci. 2007;121:1125-31.

78. Stafford JM, Raybuck JD, Ryabinin AE, Lattal KM. Increasing histone acetylation in the hippocampus-infralimbic network enhances fear extinction. Biol Psychiatry. 2012;72:25-33

79. Carrer A, Parris JL, Trefely S, Henry RA, Montgomery DC, Torres A, et al. Impact of a high-fat diet on tissue Acyl-CoA and histone acetylation levels. J Biol Chem. 2017;292:3312-22.

80. Krautkramer KA, Kreznar JH, Romano KA, Vivas El, Barrett-Wilt GA, Rabaglia ME, et al. Diet-microbiota interactions mediate global epigenetic programming in multiple host tissues. Mol Cell 2016;64:982-92.

81. Intlekofer KA, Berchtold NC, Malvaez M, Carlos AJ, McQuown SC, Cunningham MJ, et al. Exercise and sodium butyrate transform a subthreshold learning event into long-term memory via a brain-derived neurotrophic factor-dependent mechanism. Neuropsychopharmacology. 2013;38:2027-34

82. Barichello T, Generoso JS, Simoes LR, Faller CJ, Ceretta RA, Petronilho F, et al. Sodium butyrate prevents memory impairment by re-establishing BDNF and GDNF expression in experimental pneumococcal meningitis. Mol Neurobiol. 2015;52:734-40.

83. Harris AP, Lennen RJ, Brydges NM, Jansen MA, Pernet CR, Whalley HC, et al. The role of brain-derived neurotrophic factor in learned fear processing: an awake rat fMRI study. Genes Brain Behav. 2016;15:221-30.

84. Sandberg JC, Bjorck IM, Nilsson AC. Rye-based evening meals favorably affected glucose regulation and appetite variables at the following breakfast: a randomized controlled study in healthy subjects. PLoS ONE 2016;11:e0151985.

85. Sandberg JC, Björck IME, Nilsson AC. Increased plasma brain-derived neurotrophic factor $10.5 \mathrm{~h}$ after intake of whole grain rye-based products in healthy subjects. Nutrients. 2018;10:1097. 